\title{
MILTON FRIEDMAN E A EFETIVIDADE DA POLÍTICA FISCAL ${ }^{*}$
}

\author{
Francisco Luiz C. Lopreato ${ }^{(a)(b)}$
}

RESUMO: O objetivo do artigo é discutir a visão de Milton Friedman sobre o papel da política fiscal. $\mathrm{O}$ trabalho estrutura-se a partir da discussão de quatro pontos. $\mathrm{O}$ primeiro relembra traços da trajetória do autor, desde a fase inicial em que dá relevância à política fiscal até o momento em que a renega. $\mathrm{O}$ segundo retoma questões apresentadas no tópico anterior e os argumentos usados para negar a efetividade da política fiscal. O terceiro volta-se à apresentação da hipótese da renda permanente e a sua posição contra o uso e as implicações da função consumo nos estudos de caráter keynesiano da velha síntese. O último ponto destaca a crítica contra as ações discricionárias, com ênfase na discussão dos lags existentes na política fiscal. O retorno ao autor justifica-se porque as suas ideias influenciaram o modo de a atual teoria macroeconômica dominante pensar tópicos relevantes da área fiscal.

PALAVRAS-CHAVE: História do pensamento econômico; Milton Friedman; política fiscal.

CLASSIFICAÇÃO JEL: B2; B22.

Artigo recebido em 28/09/2012 e aprovado em 22/08/2013.

a Doutor em Ciência Econômica pela Universidade Estadual de Campinas (UNICAMP) e professor do Instituto de Economia da UNICAMP. Contato: lopreato@eco.unicamp.br.

b O autor agradece os comentários dos professores Rogério de Andrade e Sérgio Prado. 


\title{
MILTON FRIEDMAN AND THE EFFECTIVENESS OF FISCAL POLICY
}

\begin{abstract}
The purpose of this paper is to discuss the thoughts of Milton Friedman on the role of fiscal policy. The discussion is structured with four topics. The first brings back parts of the author's trajectory, from his initial phase where he believes on the importance of the fiscal policy to the moment he denies it. The second talks about issues presented in the previous topic and the arguments used to deny the effectiveness of fiscal policy. The third is the presentation of the permanent income hypothesis and its position against the use and implications of the consumption function in studies of the old neoclassic synthesis. The last topic highlights the criticism against the discretionary actions, with emphasis on the lags on fiscal policy. The return to the author is justified because his ideas have influenced the way of the current dominant macroeconomic theory thinking of relevant topics on fiscal policy.
\end{abstract}

KEYWORDS: History of economic thought; Milton Friedman; fiscal policy. 


\section{INTRODUÇÃO}

O objetivo do texto é resgatar a visão de Milton Friedman sobre o papel da política fiscal. O retorno à Friedman justifica-se porque, de um lado, o autor é reconhecido como o principal adversário das ideias keynesianas da síntese neoclássica das décadas de 1950, 1960 e 1970 e, de outro, influenciou, expressivamente, o modo de a teoria macroeconômica dominante pensar tópicos relevantes da área fiscal.

Friedman considerava-se seguidor da tradição de Chicago e sua trajetória intelectual pautou-se pela defesa da proposição teórica de que as mudanças na oferta monetária têm efeitos econômicos relevantes e se constituem na principal causa das variações da renda nominal e do comportamento de longo prazo dos preços. A tese de determinação da renda pela oferta de moeda pautou o percurso teórico do autor. Os seus principais textos tornaram-se referências obrigatórias da corrente monetarista e delinearam a crítica ao papel da política fiscal na teoria keynesiana ortodoxa. Como crítico do regime de política macroeconômica proposto por esta escola de pensamento, colocou-se contra as ações discricionárias e chamou a atenção para a não efetividade do uso da política fiscal como meio de se contrapor ao ciclo econômico e buscar o pleno emprego.

O seu modo de pensar a política fiscal é definido nos marcos da estrutura teórica monetarista e o embate com os autores keynesianos, expresso no debate política monetária vs política fiscal, é um instrumento de reafirmaçãodo papel da oferta de moeda ede negaçãodo princípio da demanda efetiva na manutenção da atividade econômica e do emprego. A política fiscal aparece de forma subordinada, importante para muitas coisas, mas, no contexto macroeconômico, a sua relevância está diretamente atrelada ao impacto provocado na expansão monetária, já que, por si só, não tem efeito na renda nominal, na inflação ou nas flutuações cíclicas.

Friedman, no campo convencional,"venceu” o debate. O sucesso da visão keynesiana nas primeiras décadas após o lançamento da Teoria Geral deu lugar às críticas quanto a sua capacidade de explicar a situação de alta inflação e baixo crescimento vigente nos anos 1970. As ideias monetaristas ocuparam esse espaço e dominaram a discussão teórica. O tratamento da política fiscal na nova síntese neoclássica $(\mathrm{NSN})^{1}$ - o enfoque macroeconômico atualmente dominante - incorporou os ensinamentos monetaristas e apresenta traços marcantes das propostas do autor na área fiscal, apesar de parte de seus argumentos terem sido revistos nos modelos mais aceitos à luz das diretrizes teóricas fundamentadas na lógica das expectativas racionais e da dominância dos fundamentos microeconômicos.

1 Woodford (2009) discute a ideia da nova síntese neoclássica. 
O texto propõe-se a resgatar o olhar de Friedman sobre a política fiscal a partir da análise de quatro pontos de sua visão. O primeiro relembra traços da trajetória do autor, desde a fase inicial em que dá relevância à política fiscal até o momento em que a renega. O segundo retoma questões apresentadas no tópico anterior e os argumentos usados para negar a efetividade da política fiscal. O terceiro volta-se à apresentação da hipótese da renda permanente e a sua posição contra o uso e as implicações da função consumo nos estudos de caráter keynesiano da velha síntese. O último ponto destaca a crítica contra as ações discricionárias, com ênfase na discussão dos lags existentes na política fiscal.

\section{A POSIÇÃO DE FRIEDMAN: DA RELEVÂNCIA DA POLÍTICA FISCAL À SUA TOTAL NEGAÇÃO}

A posição de Friedman a respeito do papel da política fiscal apresentou nuances ao longo do tempo; não obstante, o autor manteve a coerência dos seus argumentos e a consistência de pontos de vista entre os trabalhos pioneiros e os da maturidade no que se refere à relevância das mudanças na quantidade de moeda - política monetária como o fator decisivo na condução das condições econômicas.

Em um de seus escritos iniciais (Friedman, 1948), ao discutir o modo de alcançar a estabilidade econômica, atribuiu lugar de destaque à política fiscal. A proposta envolveu a definição das regras de atuação de quatro elementos básicos: i) o sistema monetário e bancário; ii) os gastos governamentais em bens e serviços; iii) as transferências públicas; e iv) a estrutura tributária. A preocupação inicial voltou-se à reforma do sistema monetário e bancário, com o objetivo de estabelecer regras de conduta capazes de eliminar a capacidade dos agentes privados de criarem ou de destruírem moeda, bem como o poder da autoridade monetária de controlar discricionariamente o numerário. O controle sobre o poder do sistema privado de influenciar a quantidade de moeda ocorreria por meio da fixação de compulsório de 100\% sobre os depósitos e de restrições ao uso das operações de open market, de redesconto e de alterações das reservas bancárias, de tal modo que a função de alterar o montante de moeda caberia inteiramente ao Banco Central e se daria unicamente com base na existência de déficits ou superávits do setor público.

Os elementos atrelados à política fiscal, por sua vez, deveriam obedecer a regras estreitas e abolir as alterações discricionárias do montante de gastos, deixando ao programa de transferências sociais a tarefa deresponder automaticamente às variações do movimento cíclico da atividade econômica. 
A essência da proposta de Friedman é dotar a estrutura fiscal e a conduta do sistema monetário e bancário de normas rígidas, com o propósito de deixar a política anticíclica atuar, em resposta às alterações do nível de emprego ao longo do ciclo,por meio de variações automáticas do valor absoluto dos gastos de transferências governamentais. Esses movimentos provocariam situações de superávits ou déficits públicos, com reflexo direto na oferta de moeda e no valor da renda nominal, permitindo, assim, sustentar a estabilidade ${ }^{2}$. A ação do governo compensaria, pelo menos em parte, os outros elementos da demanda agregada e responderia por mudanças na disponibilidade de moeda na economia, produzindo efeitos anticíclicos no nível de atividade econômica.

A condução da política fiscal atrelar-se-ia à política de estabilização. O governo atuaria com dois orçamentos: o ideal, equilibrado, supondo as condições de renda e estabilidade esperadas; e o real, refletindo o quadro econômico do momento, responsável por regular o volume da oferta de moeda e garantir a política de estabilização ${ }^{3}$. $\mathrm{O}$ sistema tributário e os gastos públicos teriam de ser estruturados de modo a sustentar o orçamento equilibrado, incluindo as transferências, nos períodos em que a economia estivesse no nível esperado de renda e emprego, evitando, assim, alterações na quantidade de moeda. Quando não se verifica se o nível hipotético de renda e emprego, as transferências governamentais cresceriam automaticamente, em resposta ao aumento do desemprego, alterando o resultado fiscal e gerando déficits responsáveis por ampliar o volume de moeda que, por sua vez, teria implicações sobre a renda nominal.

A estratégia coloca a política fiscal em lugar de destaque, pois o arranjo da situação fiscal é o fator de determinação das oscilações da oferta monetária. É preciso realçar que o autor já descarta neste texto o uso das ações fiscais discricionárias de manipulação da demanda agregada como meio de alcançar a estabilização, com o argumento de que a existência de lags na política fiscal, como se discute mais adiante, gera efeitos desestabilizadores e acirra as oscilações do nível de atividade, ao invés de contribuir para suavizar o ciclo. Além disso, o texto, em linha com os estudos posteriores, segue o princípio monetarista de que a mudança da renda nominal é função da variação da

\footnotetext{
2 Segundo Friedman (1948, p. 251): "Deficits or surpluses in the government budget would be reflected dollar for dollar in changes in the quantity of money; and, conversely, the quantity of money would change only as a consequence of deficits or surpluses. A deficit means an increase in the quantity of money; a surplus, a decrease. Deficits or surpluses themselves become automatic consequences of changes in the level of business activity".

3 Segundo Friedman (1948, p. 249-250): "The government would thus keep two budgets: the stable budget, in which all figures refer to the hypothetical income, and the actual budget. The principle of balancing outlays and receipts at a hypothetical income level would be substituted for the principle of balancing actual outlays and receipts".
} 
oferta monetária e não do fato em si de o governo elevar o gasto público. Porém, a oferta monetária, diferentemente do que veio a defender mais tarde, não obedece a uma regra de crescimento estável, nem está sujeita a rígido controle; ao contrário, muda ao longo do ciclo, no rastro das alterações automáticas da política fiscal, que, deste modo, ocupam lugar de destaque no movimento da economia (Condgon, 2011).

A posição de Friedman na entrevista concedida a Snowdon e Vane em 1996 parece ser outra. Ao ser perguntado sobre o papel da política fiscal no contexto macroeconômico à luz de seus estudos sobre a função consumo e economia monetária, respondeu simplesmente: " $[n]$ one. I believe that fiscal policy will contribute most if it doesn't try to offset short-term movements in the economy. I'm expressing a minority view here but it's my belief that fiscal policy is not an effective instrument for controlling short-term movements in the economy" (Snowdon e Vane, 2005, p. 213).

A sua resposta nega a contribuição da política fiscal no curto prazo para atenuar a instabilidade do cenário macroeconômico e atribui à política monetária o domínio sobre a política fiscal, bem como se afasta da postura adotada em 1948 de imputar ao resultado fiscal a responsabilidade de regular a oferta de moeda. O autor usou dois argumentos contra a ação da política fiscal: primeiro, considera errada a visão keynesiana de que o déficit é um fator de estímulo à economia, já que o efeito negativo decorrente do financiamento do déficit elimina o ganho possível com o gasto; e, segundo, aponta a maior complexidade para realizar alterações de curto prazo na política fiscal, em comparação com a política monetária, como empecilho para o uso desse instrumento.

As duas posições, embora revelem, de fato, aspectos distintos, são coerentes e intelectualmente integradas caso se levem em conta as diferenças no financiamento do gasto público. O texto de 1948 explicita a relação direta entre a política fiscal e a variação da moeda; já no de 1996, o autor trata da política fiscal por si só, sem alterações da oferta monetária, daí negar qualquer efeito sobre a renda.

O Friedman de 1948, mesmo dando destaque à ação da política fiscal, salientou a função da oferta de moeda como o fator de determinação da renda, estabelecendo, assim, a coerência dos argumentos e o elo teórico entre as posições de juventude e de maturidade. A aproximação entre as duas situações é reforçada pelo posicionamento de 1948 contra as medidas fiscais discricionárias, já que, no seu entender, os lags associados às decisões de política fiscal acabavam por gerar efeitos desestabilizadores e nada contribuíam para estabilizar a economia, como imaginavam os defensores da síntese neoclássica. 


\section{A NÃO EFETIVIDADE DA POLÍTICA FISCAL}

O embate entre Friedman e os keynesianos ortodoxos em torno da política fiscal vs política monetária reflete a disputa teórica, em um plano mais geral, sobre o sentido de determinação da renda e expõe as diferenças no modo de pensar a dinâmica econômica e o uso dos instrumentos de política econômica. A crítica à política fiscal ancora-se no princípio monetarista fundamental de que a renda nominal e os preços são funções da oferta monetária e não do volume da demanda agregada. Em consequência, a política fiscal só é efetiva quando acompanhada de variações na quantidade de moeda, caso contrário os possíveis efeitos de curto prazo dos gastos iriam se anular no decorrer do tempo.

$\mathrm{O}$ autor preocupou-se fundamentalmente em construir o seu aparato analítico e fixar a política monetária, por meio do controle da oferta de moeda, como o fator relevante, embora não único, na determinação das variações da renda e dos preços. Em alguns dos seus trabalhos mais expressivos, consolidou em termos metodológicos e empíricos a sua hipótese teórica e se utilizou dela como elemento nuclear de crítica ao pensamento keynesiano.

O texto The Quantity Theory of Money: A Restatement (1956) consolidou a base teórica do monetarismo. Friedman reafirmou a importância da teoria quantitativa da moeda, mas a reinterpretou como uma teoria da demanda de moeda, definida como relação funcional estável entre o montante demandado de moeda e pequeno número de variáveis determinantes. A hipótese de que a demanda e a velocidade de circulação da moeda são estáveis o levou a sustentar teoricamente apresença de uma relação causal e previsível entre as variações da oferta de moeda e a renda nominal, delineando o campo de investigação da teoria monetarista. Em The Quantity Theory of Money (1968a) confirmou o seu princípio básico:

It is clear from this that changes in prices and nominal income can be produced either by changes in the real balances that people wish to hold or by changes in the nominal balanced available for them to hold. Indeed it is a tautology, summarized in the famous quantity equation that all changes in nominal income can be attributed to one or the other - just as a change in the price of any good can always be attributed to a change in either demand or supply. The quantity theory is not, however, this tautology. It is rather, the empirical generalization that changes in desired real balances (in the demand for money) tend to proceed slowly and gradually or to be the result of events set in train by prior changes in supply, whereas, in contrast, substantial changes in the supply of nominal balances can and frequently occur independently any change in demand. The conclusion is that substantial changes in prices or nominal income are almost invariably the result of changes in the nominal supply of money. (p. 39) 
O passo seguinte, delineada a hipótese teórica, consistiu em realizarestudos empíricos de comprovação do princípio basilar do monetarismo. Friedman e Meiselman (1963), contrariando os pensadores keynesianos ortodoxos, reafirmaram a tese de estabilidade da velocidade de circulação da moeda e a proposição de que a política monetária, por meio de alterações na oferta de moeda, é mais responsável pelas mudanças no estoque de moeda do que as variações na demanda do público. Friedman e Schwartz, em Money and Business Cycles (1963), centraram o estudo nas causas do ciclo econômico e verificaram que a variação da oferta de moeda é consistente com o comportamento cíclico da economia. Além disso, constataram que, mantido constante o estoque de moeda, não há relação sistemática dos gastos autônomos, de investimento e consumo, como movimento cíclico da economia ${ }^{4}$ Em outro estudo clássico, A Monetary History of the United States 1867-1960 (1963), esses autores ampliaram a evidência sobre a presença da relação empírica, em contextos institucionais distintos, das variações entre a oferta de moeda, a renda nacional e o nível de preços, de modo a reafirmar a supremacia da política monetária na determinação dos movimentos da economia.

Os estudos empíricos destacaram as mudanças na taxa de expansão do estoque de moeda como condição necessária e suficiente para gerar alterações significativas na taxa de crescimento da renda nominal e firmaram a proposição basilar da crítica à efetividade da política fiscal. A proposta implica em aceitar a tese de que o resultado do orçamento governamental (déficit ou superávit) não tem efeito direto na renda, já que o seu impacto depende crucialmente da forma como ocorre o financiamento, se por empréstimo ou ampliação do estoque de moeda. Assim, a política fiscal, nessa perspectiva analítica, não é capaz, por si só, de desempenhar papel efetivo na variação da renda, o que alça as ações da política monetária à condição de instrumento responsável pela política de estabilização da economia.

Friedman, preso a esta proposição de caráter geral, aprofundou a crítica à lógica da argumentação da síntese neoclássica, detalhando, em distintas condições de financiamento, o efeito do gasto público e a consequência do crowding out. No caso de o déficit

\footnotetext{
4 Segundo Friedman e Schwartz (1963, p. 63): "These results are striking because they contradict so sharply the widespread presumption among economists that investment (or, more generally, autonomous expenditures) is the prime mover in cyclical fluctuations, transmitting its influence to the rest of income via a multiplier effect on consumption. So far as these results go, they suggest that, for a given stock of money, there is no systematic relation at all between autonomous expenditures and consumption- in experience, the multiplier effect on consumption is as likely to be negative as positive". "(...) For major movements in income, we concluded that there is an extremely strong case for the proposition that sizable changes in the rate of change in the money stock are a necessary and sufficient condition for sizable changes in the rate of change in money income" (ibidem, p. 48).
} 
ser financiado por meio de empréstimos, sem aumento do estoque de moeda, o efeito expansionista provocado pelo aumento do gasto público será compensado, em grande parte ou totalmente, pelo efeito indireto da transferência de fundos privados para o setor público. Como o estoque de moeda continua o mesmo, a maior demanda de crédito provoca um aumento na taxa de juros, que deverá subir o bastante até o ponto em que a queda do investimento privado (crowding out) compense o aumente da despesa pública. Se o governo incorrer em superávit, a sua demanda de crédito cai e cresce a disponibilidade de recursos para empréstimos ao setor privado, provocando a queda da taxa de juros e o aumento dos investimentos ${ }^{5}$. Entretanto, na hipótese de o déficit fiscal causar expansão monetária, o efeito expansionista da renda é duradouro, já que o efeito positivo do gasto público não será compensado pela redução do investimento e iráse sustentar enquanto o estoque de moeda não for ajustado (Friedman, 1962, 1968a, 1968b, 1972; Friedman e Heller, 1969).

$\mathrm{O}$ argumento desdobra-se em outras questões. A primeira refere-se à diferença de duração temporal dos efeitos do déficit público em condições distintas de financiamento. O déficit, se não alterar o volume de moeda, afeta, primariamente, a renda só durante o tempo em que ocorrer, cessando o seu efeito tão logo seja eliminado. Porém, na hipótese de acontecer a expansão monetária, o efeito positivo persiste mesmo depois de retornar o equilíbrio fiscal, pois, em termos do modelo IS-LM, há, inicialmente, o deslocamento da IS e da LM. Com a reversão do déficit, a IS volta à posição original, mas a LM não, continuando a produzir efeitos ${ }^{6}$.

A segunda questão visa criticar o uso das políticas de corte de gastos públicos ou de aumento de impostos, sem alterar o volume de moeda, com o objetivo de estabilizar a economia. Friedman acredita que o aumento dos impostos e a redução do gasto público não se colocam como meio de alcançar a estabilidade. O efeito inicial causado por esses dois movimentos revela apenas parte dos fatos. No caso de o governo resolver

\footnotetext{
Segundo Friedman e Heller (1969, p. 50): "The state of the government budget has a conservable effect on interest rate. If the federal government runs a large deficit, that means the government has to borrow in the market, which raises the demand for loanable funds and so tends to raise interest rates. If the government budget shifts to a surplus, that adds to the supply of loanable funds, which tends to lower interest rates".

6 Segundo Friedman (1972, p. 916): "We may put this point differently. Assume a one-year increase in the deficit, with the budget then returning to its initial position. If this is financed by borrowing from the public with no change in monetary growth, then, in the most rigid Keynesian system, the IS curve moves to the right and then back again; real and nominal income rise for one year, then return to their initial values. If the oneyear increase in the deficit is financed by creating money, the LM curve moves to the right as well, and stays there after the IS curve returns to its initial position. If prices remain constant, real and nominal income stay at a higher level indefinitely. If, as is more reasonable, prices ultimately rise, real income may return to its initial level, but nominal income will stay at a higher level indefinitely".
} 
cortar o gasto público ou elevar os impostos mantendo constante o montante de despesas, reduziráa demanda por novos financiamentos e deixará maior volume de recursos disponíveis nas mãos dos emprestadores. O resultado final será, então, a queda da taxa de juros e o aumento dos investimentos privados, recompondo, assim, o valor da renda. $\mathrm{Na}$ análise da política de corte de impostos vale o mesmo raciocínio e o efeito é semelhante. Segundo o autor, não está demonstrado empiricamente que a opção de cortar impostos, mantendo constante o estoque de moeda, tem, de fato, efeito expansionista sobre a renda nominal. Ademais, como será visto a seguir, segundo a hipótese de renda permanente, a medida, caso não seja entendida como duradoura, não afetará o consumo e, portanto, a renda. Ou seja, na visão de Friedman, a política fiscal, por si só, não tem efeito sobre a renda independentemente do instrumento fiscal utilizado. $\mathrm{O}$ autor lembra, no entanto, que se for para adotar uma política de caráter "fiscalista" prefere a alternativa de reduzir os gastos ao invés de elevar os impostos (Friedman e Heller, 1969).

Além disso, o autor critica a ideia keynesiana amplamente aceita de que o aumento das despesas públicas leva necessariamente à expansão da renda e o corte de gastos à retração. No seu entender, a questão, embora aparentemente simples e atraente, desconsidera os efeitos do aumento do gasto público nas decisões privadas e, consequentemente, no multiplicador. No caso de se levar em conta os efeitos nas expectativas dos agentes e na distribuição da despesa, o efeito final torna-se muito mais incerto. Pode ocorrer tanto redução da despesa, de modo que o aumento do gasto público é compensado pela queda do gasto privado e a renda nominal não se altera, até a situação prevista na visão keynesiana de elevação plena da renda. Ou ainda, simplesmente, há aumento da renda nominal, graças à elevação dos preços, com a renda real permanecendo constante ou crescendo pouco (Friedman, 1962) ${ }^{7}$.

A conclusão é simples. Friedman realça a relevância da política fiscal para muitas coisas, já que a condição do orçamento determina o nível dos impostos e a parcela da renda nacional gasta pelo setor público e pelo setor privado, além de ter efeito considerável sobre a taxa de juros. Porém, não crê na sua efetividade, qualquer que seja o tipo de política fiscal empregada, como fator de determinação da renda se não ocorrer, simultaneamente, alteração na quantidade de moeda, pois a oferta de moeda é a prin-

\footnotetext{
Friedman, depois de exemplificar o efeito do multiplicador keynesiano provocado pelo aumento de $\$ 100$ de gasto público mantido constante o nível de impostos, afirma: " $t]$ his simple analysis is extremely appealing. But the appeal is spurious and arises from neglecting other relevant effects of the change in question. When these are taken into account, the final result is much more dubious: it may be anything from no change in income at all, in which case private expenditures will go down by the $\$ 100$ by which government expenditures go up, to the full increase specified. And even if money income increases, prices may rise, so real income will increase less or not at all" (1962, p. 70).
} 
cipal responsável pela variação da renda, com efeito real no curto prazo. Como o autor afirma: "I believe that the rate of change of the money supply by itself has a very important effect on nominal income and prices in the long run. It has a very important effect on fluctuations in nominal and real income in the short run" (Friedman e Heller, 1969, p. 50).

\section{A HIPÓTESE DA RENDA PERMANENTE E A CRÍTICA À FUNÇÃO CONSUMO}

O questionamento da função consumo dos modelos keynesianos é outro elemento central da crítica de Friedman à política fiscal. A hipótese da renda permanente (Friedman, 1957) nega o conceito de Keynes de relacionar o consumo diretamente ao valor da renda corrente e trabalha com a ideia de que o consumo é definido a partir do que se espera ser a renda permanente de longo prazo, enquanto que as mudanças transitórias da renda, desde que não sejam entendidas como duradouras, afetam primariamente o valor dos ativos e das obrigações; ou seja, os seus efeitos mais significativos influenciam o montante de poupança e não o gasto de consumo.

Friedman sintetiza a função consumo em três equações básicas e aponta que a renda e o consumo são compostas de uma parte permanente e outra transitória, com lógicas de comportamento diferenciadas:

$$
\begin{aligned}
& c p=k(i, w, u) y p \\
& y=y p+y t \mathrm{e} \\
& c=c p+c t .
\end{aligned}
$$

A equação (i) define o consumo permanente (cp) como uma fração $(k)$ da renda permanente $(y p)$ - a que reflete o efeito dos fatores que determinam o valor do capital ou riqueza, considerados como a riqueza não humana e atributos pessoais -, cujo valor não está associado ao tamanho de $y p$, mas ao de outras variáveis: a) a taxa de juros $(i)$; b) a participação da riqueza não humana na renda $(w)$, e c) outros fatores (u) que refletem os gostos e preferências dos consumidores, responsáveis por influenciar as decisões sobre consumir ou acumular ativos, como a incerteza sobre o comportamento da renda, a idade, composição e efeitos associados a fatores culturais como raça e origem. Já as equações (ii) e (iii) apresentam a renda (y) e o consumo (c) como compostos pela soma de dois componentes: (1) o permanente, cuja dinâmica é explicada na equação (i) e (2) a parte transitória que reflete a influência de fatores vistos como ocasionais ou aleatórios pelos consumidores (Friedman, 1957, caps. III e IX).

O autor atribui o erro da proposta de Keynes à má especificação da função consumo. Crê que o consumo é largamente determinado por rendas passadas, atreladas a movimentos de longo prazo, e que, em sua maior parte, deve ser considerado como 
gasto autônomo, desvinculado das alterações da renda corrente oriundas da variação do gasto público ou de mudanças temporárias no montante da renda disponível. Com base nessa conceituação teórica, criticou a relevância das relações gasto-renda presa às flutuações da atividade econômica na visão keynesiana. Primeiro, questionou os pressupostos da tese de estagnação secular (Hansen, 1947) e as propostas de como enfrentar o problema baseadas no uso de impostos sobre a classe de alta renda e de transferências aos de maior propensão a consumir. Acreditava que não há razão para se esperar que o aumento do nível de renda leve a um aumento da taxa de poupança e reduza as oportunidades de investimentos, como previsto na tese de estagnação secular, nem que alterações temporárias na tributação alterassem as decisões de consumo. Segundo, argumentou que a hipótese da renda permanente interfere diretamente no cálculo da propensão marginal a consumir e também no tamanho e na estrutura no tempo do multiplicador da política fiscal, influindo, como coloca Sargent (1987), na formulação, estimação e simulação dos modelos macroeconométricos.

O multiplicador do investimento seria, então, menor do que se supõe e o sistema seria mais estável, contrariando as previsões dos modelos da síntese neoclássica de comportamento da renda. Nas palavras de Friedman (1957, p. 238):

The effect is almost certain to be a much smaller estimate of the marginal propensity to consume out of current income than would be obtained from a function that makes consumption dependent on current income alone. To put it in other terms: it means that a much larger part of current consumption is interpreted as autonomous and a much smaller part as dependent on current income and hence, through the multiplier process, on investment. The result is a smaller investment multiplier, and an inherently cyclically more stable system.

Isto é, a força da política fiscal compensatória de caráter keynesiano, segundo Friedman e Becker (1957), fundamentava-se em premissas falsas, já que os erros de especificação da função consumo induziam a erros significativos nas estimativas de renda usadas como base na construção dos modelos de apoio às políticas intervencionistas ${ }^{8}$.

8 Segundo Friedman e Becker (1957, p. 74), "[r]egarding investment as having no multiplier effects is a much better first approximation than regarding investment as the prime mover, at least with respect to the changes in real per capita income from 1905 to 1951... The finding that consumption can be predicted statistically from current income alone with a relatively small error reinforced the view that the corresponding simple Keynesian models incorporated a basically correct specification of the economic structure, although one that was unduly simple. If our analysis is valid, this widespread belief that consumption and income can be regarded as dancing primarily to the tune of unstable investment is largely the product of a statistical illusion". 
A hipótese da renda permanente também deu suporte à crítica das teorias de crescimento de cunho neoclássico que via a desigualdade na distribuição de renda como fator de determinação da taxa de poupança e propunha o uso de medidas fiscais para alterar a taxa de poupança e elevar a formação de capital ${ }^{9}$. A proposição de Friedman (1957, cap. IX) é que o efeito da desigualdade de renda depende criticamente de sua origem. Se a desigualdade advier da renda permanente, não tem efeito sobre a taxa de poupança. Por outro lado, se for o resultado de mudanças da renda transitória, a incerteza sobre o valor da renda prospectiva provoca a elevação da poupança. Ou seja, a maior taxa de poupança não decorre da desigualdade de renda em si, mas da incerteza em relação à expansão da renda permanente, que é o fator responsável por elevar a propensão a consumir.

A redução da desigualdade da renda permanente, apesar de relevante para outras questões, é neutra em relação ao valor da poupança. O que pode favorecer o aumento da taxa de poupança é a possibilidade de alterações de status social, com riscos de perdas ou ganhos, que são responsáveis por ampliar o grau de incerteza dos agentes e a disposição de eles elevarem a taxa de poupança.

\section{CRÍTICA ÀS POLÍTICAS DISCRICIONÁRIAS}

A preocupação em negar o efeito das políticas discricionárias é recorrente na obra de Friedman e já descartou o seu uso no texto de 1948, defendendo uma política anticíclica baseada em respostas da oferta de moeda às variações automáticas do resultado fiscal decorrentes das oscilações da atividade econômica. O aumento (redução) da renda, definidos o sistema tributário, o valor das transferências e o programa de gastos públicos, seria responsável por elevar (reduzir) a arrecadação, gerar um superávit (déficit) fiscal e provocar a contração (expansão) da oferta de moeda, cujas implicações sobre a demanda e o movimento da renda contribuiriam para manter a estabilidade.

As contribuições posteriores deram tratamento diferente à relação entre política fiscal e monetária constante no texto de 1948, mas mantiveram a crítica contra a ideia

9 Segundo Friedman (1957, p. 234-235): “[t]hanks to the widespread acceptance not only of the absolute income hypothesis but of a very special form of it, the inequality of the distribution of income has been regarded as a major factor explaining the aggregate savings ratio. Wide inequality of income is thought to tend toward a high savings ratio, and an approach toward equality, toward a low savings ratio. This consideration has frequently been a major argument offered in defense of inequality by people who are in other respects egalitarians: inequality in an underdeveloped country, they say, is a necessary evil since there, is no other way to generate the savings needed for economic development". 
do fine-tuning de sabor keynesiano. O autor apontou a insuficiência do conhecimento sobre os efeitos das políticas fiscal e monetária para justificar o porquê de não se adotar a prática de ajustar a política de gasto público e/ou as alíquotas dos tributos à conjuntura econômica como meio de combate aos movimentos cíclicos da economia. A manipulação da política monetária, no seu entender, responde aos objetivos de crescimento de longo prazo e serve de instrumento de combate às crises, devido ao fato de existir estreita relação entre as alterações da quantidade de moeda e da renda nacional (Laidler, 2012). Entretanto, a limitação do conhecimento dos efeitos das políticas discricionárias usadas contra as flutuações de curto prazo e o pouco que se sabe sobre as implicações de eventual mudança na quantidade de moeda nos próximos dois ou três trimestres não deixam espaço às ações discricionárias.

A proposta de Friedman, em contraste com o que defendeu em 1948, é a adoção de regra monetária explícita: a autoridade monetária não deve alterar a quantidade de moeda ao sabor da conjuntura; ao contrário, deve definir uma taxa estável de expansão do estoque de moeda. Nos anos 1980, o autor, embora convencido de que é desejável a adoção de uma regra capaz de garantir um padrão previsível de crescimento de longo prazo dos agregados monetários, passou a aceitar medidas de maior controle monetário, como meio de evitar instabilidades e afastar qualquer ameaça de inflação (Friedman 1984, 1987). Propôs, então, realizar reformas com o objetivo de: "abolish the moneycreating powers of the Federal Reserve, freeze the quantity of high-powered money, and deregulate the financial system" (Friedman, 1984, p. 24).

As colocações anteriores implicam em negar a possibilidade de a autoridade fiscal contar com a expansão monetária como meio de financiar o déficit público, criando, assim, o alicerce à estabilidade ${ }^{10}$. A contrapartida da regra monetária no plano fiscal seria planejar os investimentos e os gastos públicos de acordo com o que deseja a sociedade e definir o sistema tributário para atender a estes gastos, sem se preocupar em responder às oscilações de curto prazo da atividade econômica (Friedman, 1962, 1968).

$\mathrm{O}$ argumento do autor é o de que os defensores das medidas discricionárias não reconhecem problemas na efetividade das ações anticíclicas e não trabalham com a hipótese de que a adoção dessas práticas pode introduzir mais instabilidades do que contribuir para a sua eliminação. Segundo Friedman, os modelos a favor das ações

\footnotetext{
${ }^{10}$ Para Sargent (1987, p.9): "[i]n 1960, he advocated that the Federal Reserve adopt a rule of increasing high powered money by a constant $k$-percent a year (where $k$ is a small number to accommodate growth in the economy). This rule has the effect ofvirtually permanently denying the fiscal authorities access to the printing press and is feasible only if the federal budget is close enough to balance in a present value sense. If committed to, the $k$-percent rule has the effect of forcing the fiscal authority to finance current deficits only by credibly promising future surpluses".
} 
discricionárias assumem, implicitamente, que os efeitos das alterações dos gastos governamentais atuam como meio de se contrapor à queda da renda e não estão sujeitos a lags significativos (Friedman, 1953, 1962). Ele, ao contrário, acredita na presença de lags de três tipos: i) o tempo entre a necessidade de ação e o seu reconhecimento; ii) a demora entre o reconhecimento da necessidade de ação até a ação efetiva; e iii) o tempo decorrido desde a ação até os seus efeitos.

A existência destes lags pode levar os efeitos da ação discricionária a não ser àqueles inicialmente esperados. Os dois primeiros fatores ainda estão sujeitos a algum tipo de controle por parte dos gestores de política econômica. O último, no entanto, está atrelado às características de cada sistema econômico e o tempo transcorrido é variável e pouco previsível, podendo, de acordo com o autor, ser longo o bastante a ponto de os efeitos positivos ocorrerem não no momento do declínio da renda, mas quando já estiver em curso o movimento de recuperação, contribuindo para ampliar a instabilidade e acelerar o ciclo ao invés de minimizá-1o ${ }^{11}$.

Friedman lembra ainda que a magnitude dos efeitos da ação discricionária, embora se espere que varie em geral com o tamanho do estímulo inicial, pode ser afetada por outros fatores, como o impacto sobre a confiança dos agentes, ou não alterar o consumo, como defende a hipótese da renda permanente. Assim, o resultado não é necessariamente proporcional ao tamanho do estímulo ou na mesma direção, sendo possível pensar em um valor de gasto a partir do qual o efeito na economia não é o esperado epode chegar até mesmo a ser negativo.

A proposição tem o objetivo demostrar que o melhor a fazer é manter a estabilidade e não buscar meios de compensar as oscilações cíclicas. Isso implica em descartar o uso, de caráter keynesiano, da política discricionária: "the attempt to adapt the magnitude of government operations to the requirements of stability may therefore easily introduce more instability than it corrects" (Friedman, 1948, p. 252). A melhor opção é estabelecer regras de atuação da política fiscal, definindo o programa de investimento, o volume de impostos e o total das despesas, independentemente do ciclo econômico. Além disso, devem-se evitar mudanças compensatórias erráticas, de modo que os efeitos automáticos relacionados ao ciclo, apesar de não se saber ao certo o seu alcance, possam contribuir para a estabilidade econômica.

\footnotetext{
${ }^{11}$ Segundo Friedman (1948, p. 254): "[ $\left.t\right]$ he lag between the creation of a government deficit and its effects on the behavior of consumers and producers could conceivably be so long and variable that the stimulating effects of the deficit were often operative only after other factors had already brought about a recovery rather than when the initial decline was in progress".
} 


\section{CONSIDERAÇÕES FINAIS}

O objetivo do texto foi recuperar a visão de Milton Friedman sobre o papel da política fiscal. A questão fiscal ocupa lugar subordinado em sua obra, focada, basicamente, na defesa da política monetária como instrumento de gestão da demanda agregada e de determinação da renda e do emprego. A sua análise da política monetária, no entanto, contribuiu, de modo decisivo, para a críticado papel proeminente que a política fiscal ocupa na teoria macroeconômica de inspiração keynesiana.

$\mathrm{O}$ autor tornou-se referência obrigatória na trajetória teórica que levou a política fiscal a transitar de uma posição privilegiada após a publicação da Teoria Geral à situação vigente na concepção da NSN de quase total abandono como instrumento de administração da demanda agregada, em favor da política monetária ${ }^{12}$.

O embate de Friedman com o pensamento da síntese neoclássica colocou em xeque a efetividade da política fiscal e as suas sugestões serviram para atacar a política keynesiana. Os argumentos, baseados na hipótese da renda permanente, contrários à visão dominante sobre a dinâmica do consumo e o tamanho do multiplicador, ao lado das proposições de que a política de gastos públicos estava sujeita a lags e gerava o efeito de crowding out, serviram de apoio para questionar os fundamentos básicos da política discricionária de caráter keynesiano. E destacaram o fato de o efeito da política fiscal, por si só, não ser efetiva na determinação da renda e do emprego caso não fosse acompanhada de alterações na oferta de moeda.

As suas propostas sobre os efeitos negativos das políticas discricionárias, a presença de lags na política fiscal e a defesa de estabilizadores automáticos incorporaram-se ao pensamento da atual teoria macroeconômica dominante, bem como a ideia de que o uso de regras favorece a defesa da estabilidade. A hipótese da renda permanente ganhou aceitação ampla e inspirou, ao lado da teoria do ciclo de vida de Modigliani, o tratamento vigente da função consumo ${ }^{13}$, baseado em expectativas racionais e no pressuposto microeconômico de otimização dos agentes, ao mesmo tempo em que deu

\footnotetext{
12 A grande crise de 2008 afetou parcialmente o consenso em torno do papel quase irrelevante da política fiscal como instrumento de gestão da demanda agregada. O efeito da crise e a perda de eficácia da política monetária com taxa de juros próxima a zero reacenderam as discussões sobre a questão, mas, até o momento, pouco se avançou no sentido da ortodoxia aceitar o papel ativo da política fiscal, a não ser em condições específicas e, de preferência, com o uso dos estabilizadores automáticos.

${ }_{13}$ Segundo Hetzel (2007, p. 21): "Friedman's formulation of the permanent income hypothesis made him a pioneer in development of the optimizing framework that is the basis for modern macroeconomics".
} 
suporte teórico ao desenvolvimento da proposição de tax smoothing (Barro, 1979) ${ }^{14}$. Por outro lado, a questão da renda permanente, e a discussão sobre o efeito da política fiscal, considerando as diferentes formas de financiamento, influenciaram o campo teórico em que surgiu o princípio da equivalência ricardiana; segundo a qual o gasto público, independentemente de como é financiado - dívida ou tributos -, não tem qualquer efeito sobre a demanda global ou a taxa de juros. Ou seja, a proposição levou ao extremo a posição de Friedman contrária à efetividade da política fiscal (Barro, 1974).

Além disso, a preocupação do autor em fixar regras de coordenação das políticas fiscal e monetária colocou o controle das contas públicas como instrumento decisivo da política de estabilização e levou a outros desdobramentos teóricos novos clássicos, incorporados, posteriormente, à visão da NSN. O objetivo dos monetaristas de conter o déficit público e o financiamento inflacionário se transmudou nos modelos da NSN. A condição de estabilidade agora requer o ajuste intertemporal das contas públicas e a não presença de dominância fiscal (Sargent e Wallace, 1981), bem como o comprometimento com regras de conduta, de modo a negar as medidas discricionárias e evitar os problemas de inconsistência temporal (Kydland e Prescott, 1977).

Enfim, a NSN, mesmo se valendo de novos pressupostos teóricos, incorporou os elementos e levou adiante o embate de Friedman contra a tradição da política fiscal keynesiana da velha síntese neoclássica. A NSN abandonou a hipótese básica do monetarismo de que as oscilações econômicas podem largamente ser atribuídas às variações exógenas da oferta de moeda, passando a considerar a oferta de moeda como endógena e a creditar a responsabilidade pelas flutuações do emprego e da renda aos choques reais (mudanças tecnológicas ou nas preferências dos agentes ou mesmo alterações nas políticas governamentais e choques fiscais). O controle da oferta monetária deixou de ser visto como o instrumento básico de política monetária e surgiu no seu lugar a manipulação da taxa de juros de curto prazo, pensada, com base no conceito wickselliano de taxa de juros natural, como aquela capaz de ajustar o produto efetivo ao produto potencial, de controlar a inflação e de criar as condições de estabilidade econômica. Porém, mesmo tendo se afastado das proposições básicas do monetarismo, incorporou as ideias de Friedman sobre a política fiscal e tomou como base os seus argumentos na construção de desdobramentos teóricos, além de dar à política monetária o lugar de instrumento nuclear no combate à inflação e no ajuste da demanda agregada.

14 Como colocou Sargent (1986): "Barro's model of tax smoothing can be thought of as a reinterpretation of Milton Friedman's (1956) model of permanent income as developed by Robert E. Hall (Stochastic Implications of the Life Cycle Permanent Income Hypothesis: Theory and Evidence, 1978)". 


\section{REFERÊNCIAS BIBLIOGRÁFICAS}

BARRO, R.J. Are government bonds net wealth? J.P.E., v. 82, n. 6, p. 1095-1117, nov./dez. 1974.

BARRO, R. J. On the determination of the public debt. Journal of Political Economy, v. 87, p. 940-951, 1979.

CONGDON, T. Milton Friedman on the ineffectiveness of fiscal policy. Economic Affairs, v. 31, n. 1, p. 62-65, 2011.

FRIEDMAN, M. “Quantity theory of money”. In: EATWELL, J.; MILGATE, M.; NEWMAN, P. (Eds.) The new Palgrave: a dictionary of economics. New York: Stockton Press; and London: Macmillan, 1987, v. 4, p. 3-20. Disponível em: <http://0055d26.netsolhost.com/friedman/ pdfs/other_academia/Palgrave.1987.c.pdf>. Acesso em: 22 ago. 2013.

FRIEDMAN, M. "Monetary policy for the 1980s". In: MOORE, J. H. To promote prosperity: U.S. domestic policy in the mid-1980s. Stanford, California: Hoover Institution Press, 1984. Disponível em: <http://0055d26.netsolhost.com/friedman/pdfs/other_commentary/ Stanford.01.01.1984.b.pdf>. Acesso em: 22 ago. 2013.

FRIEDMAN, M. Comments on the critics. The Journal of Political Economy, v. 80, n. 5, p. 906950, set./out. 1972.

FRIEDMAN, M. "The quantity theory of money". In: INTERNATIONAL Encyclopedia of the Social Sciences, Free Press, 1968a, p. 432-447. Reimpresso em: WALTERS, A. A. (Ed.) Money and banking. London: Penguin Modern Economics Reading, 1973.

FRIEDMAN, M. The role of monetary policy. American Economic Review, v. 58, n. 1, p. 1-17, mar. 1968b.

FRIEDMAN, M. Capitalism and freedom. Chicago: The University of Chicago, 1962.

FRIEDMAN, M. A theory of the consumption function. Princeton: Princeton University Press, 1957.

FRIEDMAN, M. “The quantity theory of money, a restatement”. In: FRIEDMAN, M. (Ed.) Studies in the quantity theory of money. Chicago: University of Chicago Press, 1956.

FRIEDMAN, M. A monetary and fiscal framework for economic stability. The American Economic Review, v. 38, n. 3, p. 245-264, jun. 1948.

FRIEDMAN, M.; BECKER, G.A statistical illusion in judging Keynesian models. The Journal of Political Economy, v. 65, n. 1, p. 64-75, fev. 1957.

FRIEDMAN, M.; HELLER, W. W. Monetary vs. fiscal policy. New York: W. W. Norton, 1969.

FRIEDMAN, M.; MEISELMAN, D. “The relative stability of monetary velocity and the investment multiplier in the United States, 1897-1958”. In: STABILIZATION policies. Englewood Cliffs, N.J.: Prentice Hall, 1963, p. 165-268.

FRIEDMAN, M.; SCHWARTZ, A. J. Money and business cycle. The Review of Economics and Statistics, The MIT Press, v. 45, v. 1, parte 2, suplemento, p. 32-64, fev. 1963. Reimpressoem: WALTERS, A. A. (Ed.) Money and banking. London: Penguin Modern Economics Reading, 1973. 
FRIEDMAN, M.; SCHWARTZ, A. J. A monetary history of the United States: 1867-1960. Princeton: Princeton Univ. Press, 1963.

HANSEN, A. Economic policy and full employment. New York and London: McGraw-Hill, 1947.

HETZEL, R. L. The contributions of Milton Friedman to Economics. Economic Quarterly, v. 93, n. 1, p. 1-30, 2007.

LAIDLER, D. Milton Friedman's contributions to macroeconomics and their influence. Working Paper Series, Economic Policy Research Institute EPRI, n. 2012-2, fev. 2012.

KYDLAND, F. E.; PRESCOTT, E. C. Rules rather than discretion: The inconsistency of optimal plans. Journal of Political Economy, v. 85, n. 3, p. 473-491, 1977.

SARGENT, T. Interpreting the Reagan deficits. Economic Review, Federal Reserve Bank of San Francisco, n. 4, 1986.

SARGENT, T. Some of Milton Friedman's scientific contributions to macroeconomics. Stanford, CA: Hoover Institution, Stanford University, 1987.

SNOWDON, B.; VANE, H. Modern macroeconomics: its origins, development and current state. London: Edward Elgar, 2005.

WOODFORD, M. Convergence in macroeconomics: elements of the new synthesis. American Economic Journal: Macroeconomics, v. 1, n. 1, p. 267-279, jan. 2009. 
